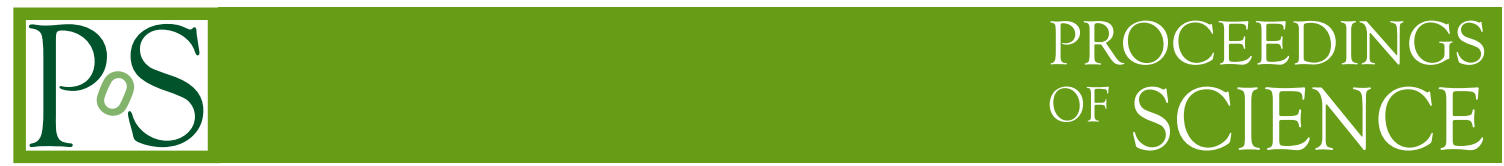

\title{
Report from the LHC Early Career Scientists Fora
}

\author{
Igor Kostiuk $^{a, b, *}$ and the LHC Early Career Scientists Fora \\ ${ }^{a}$ Nikhef, \\ Science Park 105, Amsterdam, Netherlands \\ ${ }^{b}$ Kyiv Institute for Nuclear Research, \\ prospekt Nauky 47, Kyiv, Ukraine
}

E-mail: igor.kostiuk@cern.ch

Early Career Scientists (ECSs) are non-tenured researchers. ECSs constitute nearly half of every major collaboration at the LHC and contribute to all of scientific projects and activities. This report summarizes the programs of the ECSs committees of ALICE, ATLAS, CMS, and LHCb. An overview of the joint activities of the four committees that make up the LHC Early Career Scientists Fora is given. The impact of Covid-19 on the researchers' wellbeing is discussed in conjunction with a recent survey from LHCb.

The Ninth Annual Conference on Large Hadron Collider Physics - LHCP2021

7-12 June 2021

Online

${ }^{*}$ Speaker 


\section{Introduction}

Early Career Scientists (ECSs) are defined as BSc, MSc, PhD students, and postdocs. They are researchers without a permanent contract and they make up around a half of authors in each major experiment at the LHC. Over the years ECSs self-organized and formed committees to better represent their interests, to streamline communication with the management, to establish a forum for discussion, and to provide recognition. The aforementioned committees are:

- ALICE Junior Representatives (3 members for 2 years, first elected in 2010)

- CMS Young Scientist Committee (YSC) (around 20 members with a chair, deputy and secretary for 1 year, formed in 2013)

- LHCb Early Career, Gender and Diversity Office (ECGD) (2 members for 2 years, formed in 2014, extended in 2019 by 2 ECSs)

- ATLAS Early Career Scientist Board (ECSB) (7 members for 2 years, monthly rotating chair, formed in 2017)

Efficient communication with the management of the experiments is achieved in different ways: the CMS YSC and LHCb ECGD Office have non-voting membership in the corresponding Collaboration Boards, while the ATLAS ECSB has separate meetings with the management. ALICE Junior Representatives have voting rights in the Collaboration and Management Boards. The committees advise on matters related to ECSs, and facilitate cooperation. This report provides an update on activities of the four committees. Previous updates can be found here [1][2][3].

\section{Programs by the individual committees}

The LHC Early Career Scientists Fora organizes different events which are mentioned in the next section. This has come as an evolution from numerous smaller events which were and are still organized by the individual committees. Here I provide a brief overview of those.

Each experiment has a "Welcome Event" [4][5][6][7] to introduce newcomers to the current research happening in the corresponding collaboration, to the management, project leaders, and secretariat. In addition, LHCb, ATLAS, and ALICE organize analysis software tutorials where experiment's glossary, most commonly used tools and handy resources are introduced [8]. Among the activities focused more on the social aspect are the "Meet and Eat" [9] and "Coffee With" where people who may not have met each other otherwise are paired to talk, share experiences in an informal manner. Moreover, ALICE Junior Representatives host Journal club [7] for the ECSs where the latest papers are discussed in an open manner.

Not every event is designed from the start to be repeated regularly, and in many cases a pilot is organized to measure ECSs interest. This is the case with CMS "Job Matching Event" [10] or ALICE and LHCb "Inclusive Leadership Training", which provide opportunity for ECSs, usually postdocs in junior management positions, to improve their leadership skills. Let's move on to the programs by the LHC Early Career Scientists Fora. 


\section{Programs by the LHC Early Career Scientists Fora}

Committees of the individual experiments together as the Early Career Scientists Fora organize a couple of regular events. To achieve this the committees meet twice a year (to change to 3-4 times) to share experience and to plan. Then small working groups are created to coordinate individual projects. Among those events are the "LHC Soft Skills Workshops".

The first one, titled "Working from home", was held in April 2020 [11], and since then four more have been organized [12][13], each with over 200 registrations, almost reaching 500 on two occasions [14][15]. The format is as follows: Introductory talk followed by a roundtable discussion, and a Q\&A session with all the participants. In the last one [13] we experimented with making two sessions to accomodate participants from different timezones - something that may be repeated in future.

Another example of a "one-to-many" workshop is LHC's "a conversation over ice cream". It comprises multiple thematic talks about physics, instrumentation, or software for a general audience, having a goal of introducing ECSs to topics beyond their current work. Three have been organized so far [16][17][18], and the next one has been announced [19]. Yet, some events differ in approach and follow a "one-to-one" relationship.

The first of those is "LHC Early Career Mentoring Program". The pilot started in September 2020. Mentees who are ECSs are matched with more senior scientists working for or at the LHC, preferably in a different experiment. For the first round, 22 mentors and 25 mentees were selected. They signed a confidentiality agreement and officially are in the program for nine months with the possibility of continuing to meet privately if they so choose.

Worth a separate mention is "ALICE, ATLAS, CMS and LHCb Career Networking Event". The event is organized every year by the LHC Early Career Scientists Fora together with the Alumni Network and the CERN HR department [20][21][22]. The next one is planned for November[23]. The event comprises presentations by LHC alumni who have moved out of high energy physics to other fields of science or industry, followed by a panel discussion and a Q\&A session, and provides opportunities for informal meetings and networking.

Sometimes standalone events are organized, the latest one being "Healthy Minds for Master Minds: Mental Health event for early-career scientists" [24]. It featured a professional therapist who discussed topics such as: how to recognize stress, anxiety, and depression, and how to deal with them. As a separate topic, the LHCb Mental Health survey should be mentioned.

\section{LHCb Mental Health Survey}

In December 2020 a survey on mental wellbeing among the LHCb scientists was carried out. The survey results have been subsequently published as a document [25]. In the document, outcomes of the survey regarding the social response to the Covid-19 pandemic are reported and discussed in detail. It was found that the wellbeing of ECSs has deteriorated, which is indicated by a decline in productivity, motivation, focus, and general mental health since the beginning of the pandemic. The report raises awareness about effects of the crisis and provides a number of suggestions on how to alleviate some of the issues [26]. 
The LHC Early Career Scientists Fora is determined to help ECSs during the Covid-19 pandemic by sharing tools (the aforementioned "Healthy Minds for Master Minds" event and "LHC Soft Skills Workshop: Working from home") and communicating important matters to the wider scientific community.

\section{Conclusions}

While the experiments' committees formally self-organized at different times, they have quickly converged to a number of common goals: providing representation and a forum for discussion, streamlining communication with the management, and helping with the recognition of ECSs work. Starting with smaller events in each individual experiment, and continuing in LHC-wide collaboration to organize "LHC Soft Skills Workshops", "LHC Early Career Mentoring Program", and LHC's "a conversation over ice cream" the LHC Early Career Scientists Fora is constantly looking to enhance the experience of ECSs in every possible way: by providing opportunies to improve the science-related skills, soft skills, raising awareness about wellbeing, and boosting communication. We can be reached at: lhc-early-career-scientists-fora@cern.ch

Or for the individual committees:

- ALICE Junior Representatives alice-junior-reps@cern.ch

- ATLAS Early Career Scientist Board (ECSB) atlas-ecsb@cern.ch

- CMS Young Scientist Committee (YSC) cms-young-scientist-committee@ cern.ch

- LHCb Early Career, Gender and Diversity Office (ECGD) lhcb.ecgd@ cern.ch

We are looking forward to your comments and suggestions!

\section{References}

[1] M. Muskinja, A word from the Early Career Scientists in the LHC experiments, PoS LHCP2018 (2018) 129.

[2] E.N. Umaka, Report from Young Scientist Fora at LHC, PoS LHCP2019 (2019) 074.

[3] T.A. Vami, Report from Young Scientist Fora at LHC, PoS LHCP2020 (2020) 202.

[4] "LHCb ECGD Office News Archive."

https://web.archive.org/web/20211002204009/https:

//lhcb.web.cern.ch/ECGD_Office/oldnews.html.

[5] "Blog: New ATLAS members, welcome on board."

https://web.archive.org/web/20211002211622/https:

//atlas . cern/updates/blog/new-atlas-members-welcome-board.

[6] "Induction course for newcomers in CMS."

https://web.archive.org/web/20211002212022/https:

//cms . cern/news/induction-course-newcomers-cms. 
[7] "The ALICE Newsletter No. 166."

https://web.archive.org/web/20211002211633/https:

//alice-collaboration.web.cern.ch/node/35185.

[8] “LHCb starterkit." https://web .archive.org/web/20211002212237/https:

//lhcb.github.io/starterkit/.

[9] "ATLAS: Planning an event during a pandemic."

https://web.archive.org/web/20211002212659/https:

//atlas.cern/updates/blog/ecsb-plan-during-pandemic.

[10] "CMS Job Matching Event." https://indico. cern. ch/event/1006855/.

[11] "LHC Soft skills workshop: making the best of working from home." https://indico.cern.ch/event/906623/.

[12] "LHC Soft skills workshop: Science Communication." https://indico.cern.ch/event/966250/.

[13] "LHC Soft skills workshop: Personal statement and research proposal writing." https://indico.cern.ch/event/1034518/.

[14] "LHC Soft skills workshop: CV-writing for academia and industry." https://indico.cern.ch/event/914790/.

[15] "LHC Soft skills workshop: Interview skills for academia." https://indico.cern.ch/event/1007082/.

[16] "A fresh look at the Universe: a conversation over ice cream." https://indico.cern.ch/event/737196/.

[17] "Which flavour do you want your neutrino? A conversation over ice cream." https://indico.cern.ch/event/838486/.

[18] "A conversation over ice screens: future detectors for particle physics." https://indico.cern.ch/event/914311/.

[19] "Machine learning in high energy physics: a conversation over ice cream." https://indico.cern.ch/event/1074354/.

[20] "ALICE, ATLAS, CMS and LHCb Career Networking Event 2018." https://indico.cern.ch/event/763368/.

[21] "ALICE, ATLAS, CMS and LHCb Career Networking Event 2019." https://indico.cern.ch/event/843605/.

[22] “ALICE, ATLAS, CMS and LHCb (ONLINE) Career Networking Event 2020.” https://indico.cern.ch/event/962798/. 
[23] "ALICE, ATLAS, CMS and LHCb (HYBRID) Career Networking Event 2021." https://indico.cern.ch/event/1083877/.

[24] "Healthy Minds for Master Minds: Mental Health event for early-career scientists." https://indico.cern.ch/event/1040121/.

[25] F. Dordei, E. Ben Haim, F. Cicala, S. Klaver, R. Kopecna, V. Lukashenko et al., Results of the survey on the effects of the Covid-19 pandemic on LHCb scientists, Tech. Rep., CERN, Geneva (Feb, 2021).

[26] "Survey: Covid-19 demotivates junior expat researchers most." https://web.archive.org/web/20211002214223/https://www.nikhef.nl/en/ news/survey-covid-19-is-hitting-junior-researchers-most/. 